\title{
Study of matter density distributions, elastic charge form factors and size radii for halo ${ }^{11} \mathrm{Be},{ }^{19} \mathrm{C}$ and ${ }^{11} \mathrm{Li}$ nuclei
}

\author{
Arkan R. Ridha and Zaid M. Abbas
}

Department of Physics, College of Science, University of Baghdad, Baghdad- Iraq

E-mail: zaboreshe@gmail.com

\begin{abstract}
In this work, the calculation of matter density distributions, elastic charge form factors and size radii for halo ${ }^{11} \mathrm{Be},{ }^{19} \mathrm{C}$ and ${ }^{11} \mathrm{Li}$ nuclei are calculated. Each nuclide under study are divided into two parts; one for core part and the second for halo part. The core part are studied using harmonic-oscillator radial wave functions, while the halo part are studied using the radial wave functions of Woods-Saxon potential. A very good agreement are obtained with experimental data for matter density distributions and available size radii. Besides, the quadrupole moment for ${ }^{11} \mathrm{Li}$ are generated.
\end{abstract}

Key words

Halo nuclei, matter density distributions, elastic charge form factor, matter and charge root-mean square radii, quadrupole moments.

Article info.

Received: Jun. 2017

Accepted: Sep. 2017

Published: Mar. 2018

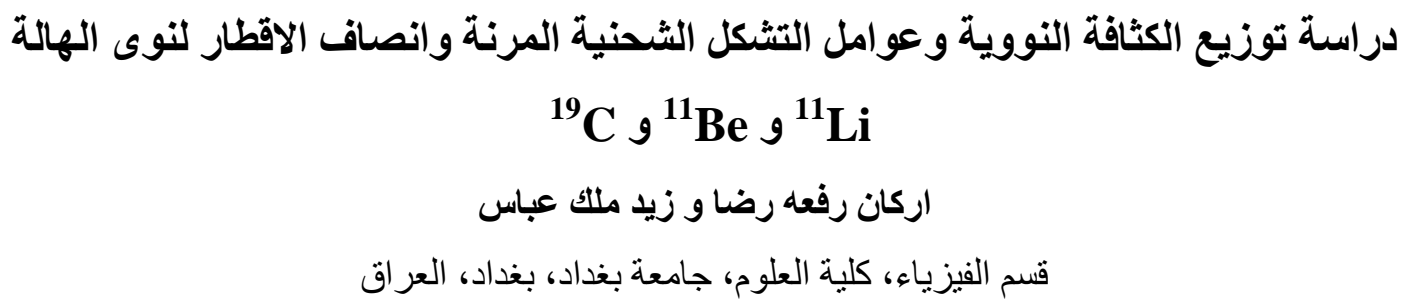

في هذا العمل، تم حساب نوزيع الكثافة النووية و عو امل التشكل المرنة وانصاف الاقطار الحجمية لنوى الهالة

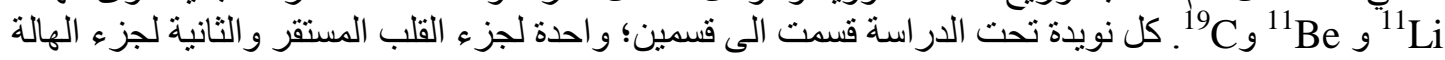

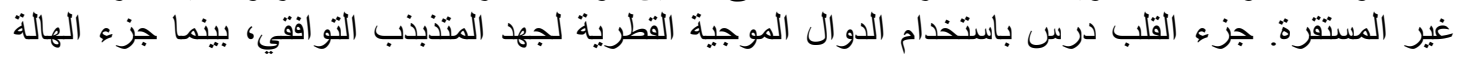

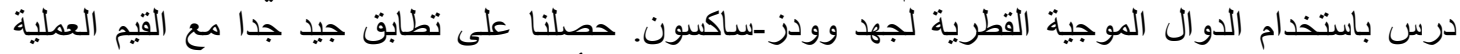

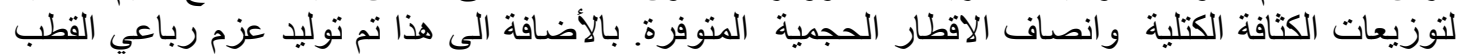

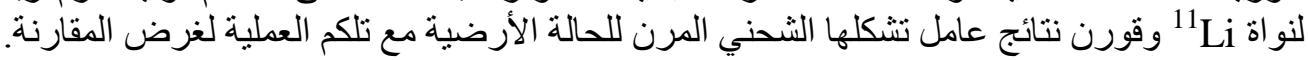

\section{Introduction}

The development of the radioactive ion beams has allowed studies of nuclei far from stability. This technical headway led to the discovery of halo nuclei on the neutron-rich side of the valley of stability $[1,2]$. These weakly bound nuclei have a strongly clusterized structure [3-6]. In a simple model, they are seen as a core that contains most of the nucleons, to which one or two neutrons are loosely bound. Due to this poor binding, the valence neutrons tunnel far outside the classically allowed region and form a sort of halo around the core [7].

The charge (nucleon) density distribution can be determined by electron (hadron) scattering experiments. Among hadron scattering, proton elastic scattering at intermediate energies besides, nucleus-nucleus scattering using Glauber models are 
good tools to probe the nucleon density [8].

Al-Khalili and Tostevin [9] re-examine the matter radii of diffuse halo nuclei as deduced from reaction cross section measurements at high energies. They took ${ }^{11} \mathrm{Li},{ }^{11} \mathrm{Be}$ and ${ }^{8} \mathrm{~B}$ as examples and showed that data require significantly larger matter radii than previously reported. Al-Khalili, and Thompson [10], studied root mean square (rms) matter radii of halo nuclei. They reviewed a basic measure in constructing, constraining, and assessing theoretical models of halo structures. They took loosely bound two- and three-body systems ${ }^{11} \mathrm{Be},{ }^{6} \mathrm{He}$, ${ }^{11} \mathrm{Li}$, and ${ }^{14} \mathrm{Be}$. Esbensen et al. [11] investigated the consistency of the measured charge radius and dipole response of ${ }^{11} \mathrm{Li}$ within a three-body model. They showed how these observables are related to the mean square distance between the ${ }^{9} \mathrm{Li}$ core and the center of mass of the two valence neutrons. Alkhazov and Sarantsev [12] cleared up the sensitivity of the nucleus-nucleus scattering to the nuclear matter distributions of exotic halo nuclei, They have calculated differential cross sections for elastic scattering of the ${ }^{6} \mathrm{He}$ and ${ }^{11} \mathrm{Li}$ nuclei on several nuclear targets at the energy of 0.8 $\mathrm{GeV} /$ nucleon with different assumed nuclear density distributions in ${ }^{6} \mathrm{He}$ and ${ }^{11} \mathrm{Li}$. Kanungo et al. [13] derived proton radii of ${ }^{12-19} \mathrm{C}$ densities from first accurate charge changing cross section measurements at $900 \mathrm{~A} \mathrm{MeV}$ with a carbon target. A thick neutron surface evolves from $\sim 0.5 \mathrm{fm}$ in ${ }^{15} \mathrm{C}$ to $\sim 1 \mathrm{fm}$ in ${ }^{19} \mathrm{C}$. The halo radius in ${ }^{19} \mathrm{C}$ is found to be $6.4 \pm 0.7 \mathrm{fm}$ as large as ${ }^{11} \mathrm{Li}$. Their $\mathrm{Ab}$ initio calculations based on chiral nucleon-nucleon and threenucleon forces reproduced well the radii. Karataglidis et al. [14] Diverse means are used to investigate ${ }^{17,19} \mathrm{C}$, their estimates have been made using a shell model for the systems. Information from those shell model studies were then used in evaluating cross sections of the scattering of $70 \mathrm{~A}$ $\mathrm{MeV}{ }^{17,19} \mathrm{C}$ ions from hydrogen.

\section{Theoretical formulations}

The ground density distributions of point neutron and proton for halo nuclei can be written as [15]:

$$
\rho_{n / p}(r)=\rho_{n / p}^{c}(r)+\rho_{n / p}^{h}(r)
$$

where the density distributions of core $\left(\rho_{n / p}^{c}(r)\right)$ are calculated using the radial wave functions $\left(R_{n l}(r)\right)$ of harmonic-oscillator (HO) potential [16] as follows:

$\rho_{n / p}^{c}(r)=\frac{1}{4 \pi} \sum_{n l} X_{n / p}^{n l, \text { core }}\left|R_{n l}\left(r, b_{t_{z}}\right)\right|^{2}$

In Eq. (2), $\quad X_{n / p}^{n l, \text { core }}$ represents the number of neutrons/protons in the $n l$ shell, where $n$ and $l$ represent the principal and orbital quantum numbers, respectively. $b_{t_{z}}$ and $t_{z}$, represent the $\mathrm{HO}$ size parameters and isospin quantum number $\left(t_{z}=\frac{1}{2}=p\right.$ for proton and $t_{z}=-\frac{1}{2}=n$ for neutron). It is worth mentioning that the summation in Eq. (2) spans all occupied orbits in the core for any nuclear sample under study. The matter density distribution for whole core nucleon can be written as a sum the densities of core neutrons and protons $\quad\left(\rho_{m}^{c}(r)=\rho_{p}^{c}(r)+\rho_{n}^{c}(r)\right)$. $\rho_{n / p}^{h}(r)$ in Eq. (1), represents the density distribution for halo part and it is calculated using Woods-Saxon (WS) radial wave functions and can be written as:

$\rho_{n / p}^{h}(r)=$

$\frac{1}{4 \pi} \sum_{n l j} X_{n / p}^{n l j, h a l o}\left|R_{n l j, t_{z}}(r)\right|^{2}$ 
$X_{n / p}^{n l j, h a l o}=N o_{t_{z}}^{\text {halo }} a_{n l j}$

In Eq. (3) and (4), No $o_{t_{z}}^{\text {halo }}$ and $X_{n / p}^{n l j, h a l o}$ represent the occupation and fractional occupation numbers of neutrons, protons, or nucleons, respectively in the sub-shell $n l j$, where $j$ represents the total angular quantum number. Besides, $a_{n l j}$ represents probability of the existence of the halo nucleons in higher sub-shells. Again, the matter density distribution for whole halo nucleons can be written as a sum the densities of halo neutrons and protons $\left(\rho_{m}^{h}(r)=\rho_{p}^{h}(r)+\rho_{n}^{h}(r)\right)$. $R_{n l j, t_{z}}(r)$ is the solution to the radial part of Schrödinger equation [17]:

$\left(\frac{\hbar^{2}}{2 \mu} \frac{d^{2}}{d r^{2}}-v(r)-\frac{l(l+1) \hbar^{2}}{2 \mu r^{2}}+\right.$

$\left.\varepsilon_{n l j, t_{z}}\right) R_{n l j, t_{z}}(r)=0$

where $\quad \mu=m_{t_{z}}(A-1) / A \quad$ is the reduced mass of the core $(A-1)$ and single nucleon, $m_{t_{z}}$ is the mass of nucleon, $A$ is the atomic mass, $\varepsilon_{n l j, t_{z}}$ is the separation energy of single halo nucleon.

The local potential $v(r)$ in Eq. (5) is taken to be WS as shown in the compact form below $[18,19]$ :

$v(r)=v_{\text {cent }}(r)+v_{\text {s.o. }}(r)+v_{c}(r)$

where

$v_{\text {cent }}(r)=\frac{-U_{0}}{\left(1+e^{\left(\frac{r-R}{a}\right)}\right)}$

represents the central part of $v(r), U_{0}$ is the strength or depth of central potential, $a$ is the diffuseness and
$R=r_{0}(A-1)^{1 / 3} \quad$ is the radius parameter.

$v_{\text {s.o. }}(r)=$

$\left(\frac{\hbar}{m_{\pi} c}\right)^{2} \frac{U_{\text {S.o. }}}{r} \frac{d}{d r} \frac{1}{\left(1+e^{\left(\frac{r-R_{\text {S.O. }}}{a_{\text {S.o. }}}\right)}\right)}\langle\hat{l} . \hat{\sigma}\rangle=$

$-\left(\frac{\hbar}{m_{\pi} c}\right)^{2} \frac{U_{\text {S.o. }}}{r} \frac{e^{\left(\frac{r-R_{\text {S.o. }}}{a_{\text {S.o. }}}\right)}}{\left(1+e^{\left(\frac{r-R_{\text {S.o. }}}{a_{\text {S.o. }}}\right)}\right)^{2}}\langle\hat{l} . \hat{\sigma}\rangle$

where $\left(\frac{\hbar c}{m_{\pi} c^{2}}\right)^{2} \approx 2 \mathrm{fm}^{2}$ with $m_{\pi} c^{2}=$ $139.567 \mathrm{MeV}$

and $\hbar c=197.329 \mathrm{MeV} . \mathrm{fm}$.

$\langle\hat{l} . \hat{\sigma}\rangle=\left\{\begin{array}{l}-\frac{1}{2}(l+1) \quad \text { for } j=l-\frac{1}{2} \\ \frac{1}{2} l \quad \text { for } j=l+\frac{1}{2}\end{array}\right.$

Eq. (8) represents the spin-orbit part of $v(r), m_{\pi}$ is the pion mass, $U_{\text {s.o. }}$ is the strength or depth of spin-orbit potential, $a_{\text {s.o. }}$ is the diffuseness of spin-orbit part, $R_{\text {s.o. }}=r_{S . O}(A-1)^{1 / 3}$ is the radius parameter of spin-orbit and $\hat{l}$ and $\hat{\sigma}$ are the angular momentum and the spin operators respectively.

Finally, in Eq. (6) $v_{c}(r)$ indicates the Coulomb potential generated by a homogeneous charged sphere and can be written as [20]:

$v_{C}(r)=$
$\left\{\begin{array}{cc}(Z-1) \frac{e^{2}}{r} & \text { if } r>R \\ \frac{(Z-1) e^{2}}{2 R}\left[3-\frac{r^{2}}{R^{2}}\right] & \text { if } r<R\end{array}\right.$,

For protons and $v_{C}(r)=0$ for neutrons, with $e^{2}=1.44 \mathrm{MeV}$. fm.

Therefore, Eq. (6) can be written as:

$v(r)=\frac{-U_{0}}{\left(1+e^{\left(\frac{r-R}{a}\right)}\right)}-2\left(\frac{\hbar}{m_{\pi}}\right)^{2} \frac{1}{r} \frac{U_{\text {S.O. }}}{a_{\text {S.O. }}} \frac{e^{\left(\frac{r-R_{S . O .}}{a_{S . O .}}\right)}}{\left(1+e^{\left(\frac{r-R_{S . O} .}{a_{S . O .}}\right)}\right)^{2}}\langle\hat{l} . \hat{\sigma}\rangle+v_{C}(r)$

The charge density distribution $\rho_{c h}(r)$ (CDD) is obtained by folding 
the proton density $\rho_{p r}$ into the distribution of the point proton density in Eq. (1) as follows [18]:

$\rho_{c h}(r)=\int \rho_{p}(r) \rho_{p r}\left(\boldsymbol{r}-\boldsymbol{r}^{\prime}\right) d \boldsymbol{r}^{\prime}$

where $\rho_{p}(\vec{r})$ is taken to have a Gaussian form, as follows [18]:

$\rho_{p r}(r)=\frac{1}{\left(\sqrt{\pi} a_{p r}\right)^{3}} e^{\left(\frac{-r^{2}}{a_{p r}^{2}}\right)}$

where $a_{p r}=0.65 \mathrm{fm}$. Such value of $a_{p r}$ reproduces the experimental charge $r m s$ radius of the proton, $\left\langle r^{2}\right\rangle_{p r}^{1 / 2}=\left(\frac{3}{2}\right)^{1 / 2} a_{p r} \approx 0.8 \mathrm{fm}$.

The ground state density distributions of point neutron and proton for stable nuclei can be written as:

$\rho_{n / p}(r)=\frac{1}{4 \pi} \sum_{n l} X_{n / p}^{n l}\left|R_{n l}(r)\right|^{2}$

The rms radii of neutron, proton, charge and matter can be directly calculated from their density distributions [18]:

$\left\langle r^{2}\right\rangle_{n, p, c h, m}^{1 / 2}=\sqrt{\frac{4 \pi}{x} \int_{0}^{\infty} \rho_{n, p, c h, m}(r) r^{2} d r}$

In Eq. (14), $X$ denotes to $N$ (number of neutrons), $Z$ (atomic number which is the same for proton and charge) and $(A)$, respectively.

The longitudinal electron scattering form factors in the first Born approximation can be written as [21, 22]:

$$
\left|F_{J}^{C}(q)\right|^{2}=\frac{4 \pi}{Z^{2}\left(2 J_{i}+1\right)}\left|\left\langle J_{f}\left\|\boldsymbol{O}_{J}^{C}(q)\right\| J_{i}\right\rangle\right|^{2}
$$

where $q$ represents the momentum transfer from electron to nucleus during scattering. Eq. (15) can be simplified to the following [23]:

$$
\left|F_{J}^{C}(q)\right|^{2}=\frac{4 \pi}{Z^{2}\left(2 J_{i}+1\right)}\left|\int_{0}^{\infty} j_{J}(q r) \rho_{c h, J}(r) r^{2} d r\right|^{2}
$$

where $j_{J}(q r)$ and $\rho_{c h, J}(r)$ are spherical Bessel function and charge transition density distribution, respectively.

The total longitudinal form factors are given by:

$|F(q)|^{2}=\sum_{J}\left|F_{J}^{C}(q)\right|^{2}$

For small $q$ leading to photon point $\left(q=w=\frac{E_{x}}{\hbar c}\right), \quad E_{x}$ is the excitation

$$
\begin{gathered}
\left|F_{J}^{C}(q=w)\right|^{2}=\frac{4 \pi}{Z^{2}\left(2 J_{i}+1\right)}\left(\frac{q^{J}}{(2 J+1) ! !}\right)\left|\int_{0}^{\infty} \rho_{c h, J}(r) r^{J+2} d r\right|^{2} \\
\left|F_{J}^{C}(q=w)\right|^{2}=\frac{4 \pi}{Z^{2}\left(2 J_{i}+1\right)}\left(\frac{q^{J}}{(2 J+1) ! !}\right)^{2}\left|\left\langle J_{f}\left\|\boldsymbol{\mathcal { O }}_{J}(\vec{r})\right\| J_{i}\right\rangle\right|^{2}
\end{gathered}
$$

From Eq. (19), the multiparticle multipole operator can be written as: reduced matrix element of electric

$$
\left\langle J_{f}\left\|\boldsymbol{O}_{J}(\vec{r})\right\| J_{i}\right\rangle=Z \frac{(2 J+1) ! !}{q^{J}} \sqrt{\frac{\left(2 J_{i}+1\right)}{4 \pi}} F_{J}^{C}(q=w)
$$

The quadrupole moment is related to the multiparticle reduced matrix element of electric multipole operator by the relation [20]: 


$$
Q=\sqrt{\frac{16 \pi}{5}}\left(\begin{array}{ccc}
J & 2 & J \\
-J & 0 & J
\end{array}\right)\left\langle J_{f}\left\|\boldsymbol{\mathcal { O }}_{J}(\vec{r})\right\| J_{i}\right\rangle
$$

Therefore, the quadrupole moment can be reduced to the following final formula:

$$
Q=\sqrt{\frac{16 \pi}{5}}\left(\begin{array}{ccc}
J & 2 & J \\
-J & 0 & J
\end{array}\right) \frac{(2 J+1) ! ! Z}{q^{J}} \sqrt{\frac{\left(2 J_{i}+1\right)}{4 \pi}} F_{J=2}^{C}(q=w)
$$

The transition density distribution in Eq. (16) are coming from contribution of core-polarization (CP) and model-space (MS) as follows [24]: $\rho_{c h, J}(r)=\rho_{c h, J}^{C P}(r)+\rho_{c h, J}^{M S}(r)$

In the present work, $\rho_{c h, J}^{C P}(r)$ is calculated using Tassie [25-27] and Bohr-Mottelson [28] models, respectively as: $\rho_{c h, J}(r)=N r^{J-1} \frac{d}{d r} \rho_{c h}(r)$

and

$\rho_{c h, J}(r)=N \frac{d}{d r} \rho_{c h}(r)$

where, $N$ in the above two equations are found so as to reproduce the experimental quadrupole moments. $\rho_{J, t_{z}}^{M S}(r)$ can be written as [29]:

$\rho_{J, t_{z}}^{M S}(r)=\frac{1}{\sqrt{4 \pi}} \frac{1}{\sqrt{2 J_{i}+1}} \sum_{a b} X_{a, b, t_{z}}^{J_{f} J_{i} J}\left\langle j_{a}\left\|Y_{J}\right\| j_{b}\right\rangle R_{n_{a} l_{a} j_{a}, t_{z}}(r) R_{n_{b} l_{b} j_{b}, t_{z}}(r)$

where $X_{a, b, t_{z}}^{J_{f} J_{i} J}$ is the proton or neutron one body density matrix element and $a$ and $b$ stand for the single-particle states.

The incoherent sum of the longitudinal form factor for ground $\mathrm{C} 0$ and $\mathrm{C} 2$ parts can be written as:

$|F(q)|^{2}=\left|F_{0}(q)\right|^{2}+\left|F_{2}(q)\right|^{2}$

The $\rho_{c h}(r)$ CDD coming from contributions of one protron in the model-space is obtained by folding the single protron charge density $\rho_{p r}$ into the distribution of the point proton density $\left(\rho_{J, p}^{M S}(r)\right)$ in Eq. (25) with the same procedure followed in Eq. (11).

The $\rho_{c h}(r)$ CDD coming from contributions of neutrons in the modelspace is obtained by folding the single neutron charge density $\rho_{\text {neu }}$ into the distribution of the point neutron density $\left(\rho_{J, n}^{M S}(r)\right)$ in Eq. (25) as follows [30]:

$\rho_{c h}(r)=\int \rho_{J, n}^{M S}(r) \rho_{\text {neu }}\left(\boldsymbol{r}-\boldsymbol{r}^{\prime}\right) d \boldsymbol{r}^{\prime}$ where $\rho_{\text {neu }}(\vec{r})$ is taken to have a Gaussian form, as follows [30]:

$\rho_{\text {neu }}(r)=\frac{1}{\left(\pi r_{i}^{2}\right)^{3 / 2}} \sum_{1}^{2} \theta_{i} e^{-r^{2} / r_{i}^{2}}$

where the parameter $\theta_{i}$ and $r_{i}$ from Chandra and Sauer are given in Table 1.

Table 1: Parameter of the neutron charge distributions.

\begin{tabular}{|c|c|}
\hline$\theta_{1}$ & 1 \\
\hline$\theta_{2}$ & -1 \\
\hline$r_{1}^{2}\left(\mathrm{fm}^{2}\right)$ & 0.469 \\
\hline$r_{2}^{2}\left(\mathrm{fm}^{2}\right)$ & 0.546 \\
\hline
\end{tabular}

For ${ }^{11} \mathrm{Li}$, the shell model calculation is done using the NuShell computer for windows [31] with the interaction psdmk [32] where the core is taken with be $\left(1 s_{1 / 2}\right)^{4}$ while the modelspace spans all psd subshells $\left(1 p_{3 / 2} 1 p_{1 / 2} 1 d_{5 / 2} 2 s_{1 / 2} 1 d_{3 / 2}\right)^{7}$. 


\section{Results and discussions}

In the present work, the MDDs, rms proton, charge, neutron, and matter radii, besides elastic electron scattering charge form factor, are computed using the radial wave functions of $\mathrm{HO}$ potential for core part for all nuclei under study, the halo part are computed using the radial wave function of WS potential. Regarding the core part, two HO size parameters are used, one for protons $\left(b_{p}\right)$ and the second for neutrons $\left(b_{n}\right)$ in order to regenerate the available experimental rms radii.

The depth of central part of WS potential $\left(U_{0}\right)$ in this work is chosen so as to reproduce the experimental single-nucleon separation energies for ${ }^{11} \mathrm{Be},{ }^{19} \mathrm{C}$ and ${ }^{11} \mathrm{Li}$ nuclei, while other parameters in Eq. (8) are fixed to be $U_{\text {s.o. }}=10, \quad a_{0}=a_{\text {s.o. }}=0.6, \quad r_{0}=$ $r_{\text {S.O. }}=R_{C}=1.2$.

For halo ${ }^{11} \mathrm{Be},{ }^{19} \mathrm{C}$ and ${ }^{11} \mathrm{Li}$ nuclei, the chosen size parameters of $\mathrm{HO}$ potential for protons and neutrons in the core part and the parameters of WS potential are presented in Table 2 . The configuration mixing included in our calculations as mentioned in Eq. (4) using probability amplitudes $\left(a_{n l j}\right)$. In this work the configuration mixing are chosen between the sub-shells, $a_{1 \mathrm{~d} 5 / 2}$, $a_{2 \mathrm{~s} 1 / 2}$ and $a_{1 \mathrm{~d} 3 / 2}$ for ${ }^{11} \mathrm{Be}$ and ${ }^{19} \mathrm{C}$, while the configuration mixing for ${ }^{11} \mathrm{Li}$ are chosen to be between $a_{1 \mathrm{p} 1 / 2}$ and $a_{2 \mathrm{~s} 1 / 2}$, as shown in Table 2 .

The computed rms proton, charge, neutron, and matter radii are presented in Table 3, for the nuclei under study. The computed results of the rms charge and matter radii are well predicted for the nuclei under study.

The calculated MDDs are shown in Fig.1 and compared with experimental data. The solid and dashed curves represent the calculated MDDs for nuclei under study using $\mathrm{HO}+\mathrm{WS}$ and $\mathrm{HO}+\mathrm{HO}$, respectively. It is obvious that the inclusion of configuration mixing to insure the contribution from higher subshells leads to freely get match between the calculated matter density distribution and experimental at tail part. Very good agreements are obtained with experimental MDDs in all figures shown in Fig. 1.

Table 2: $\mathrm{HO}$ and WS parameters for core and halo parts.

\begin{tabular}{|c|c|c|c|c|c|}
\hline nucleus & $\begin{array}{c}\text { HO size } \\
\text { parameters for } \\
\text { core }(f m)\end{array}$ & $n l_{j}$ & $\begin{array}{l}\text { Occupation } \\
\text { percentage } \\
\left(\text { per }_{n, p, m}^{n l j, h a l o}\right)^{*}\end{array}$ & $U_{0}(\mathrm{MeV})$ & $\begin{array}{c}\text { Separation } \\
\text { energies of halo } \\
\text { nucleons }(\mathrm{MeV}) \\
{[33]}\end{array}$ \\
\hline \multirow[b]{3}{*}{${ }_{4}^{11} B e_{7}$} & \multirow{3}{*}{$\begin{array}{l}b_{n}=1.660 \\
b_{p}=1.647\end{array}$} & $1 d_{5 / 2}$ & $10 \%$ & 62.933 & $S_{n}=0.50164$ \\
\hline & & $2 s_{1 / 2}$ & $70 \%$ & 62.5234 & $S_{n}=0.50164$ \\
\hline & & $1 d_{3 / 2}$ & $20 \%$ & 90.67812 & $S_{n}=0.50164$ \\
\hline \multirow{3}{*}{${ }_{6}^{19} C_{13}$} & \multirow{3}{*}{$\begin{array}{l}b_{n}=1.921 \\
b_{p}=1.631\end{array}$} & $1 d_{5 / 2}$ & $10 \%$ & 41.16488 & $S_{n}=0.580$ \\
\hline & & $2 s_{1 / 2}$ & $70 \%$ & 44.62558 & $S_{n}=0.580$ \\
\hline & & $1 d_{3 / 2}$ & $20 \%$ & 61.14195 & $S_{n}=0.580$ \\
\hline \multirow[t]{2}{*}{${ }_{3}^{11} L i_{8}$} & \multirow[t]{2}{*}{$\begin{array}{l}b_{n}=1.75 \\
b_{p}=1.727\end{array}$} & $1 p_{1 / 2}$ & $90 \%$ & 44.77765 & $\begin{array}{c}S_{2 n}=0.36928 \\
S_{n}=0.18465\end{array}$ \\
\hline & & $2 s_{1 / 2}$ & $10 \%$ & 59.82919 & $S_{n}=0.18465$ \\
\hline
\end{tabular}

$* \operatorname{per}_{n, p, m}^{n l j, \text { halo }}=\frac{X_{n, p, m}^{n l j, \text { halo }}}{N o_{t_{z}}^{\text {halo }}}=\sum_{n l j} a_{n l j}$ 
Table 3: The calculated $\left\langle r^{2}\right\rangle_{c h}^{1 / 2},\left\langle r^{2}\right\rangle_{p}^{1 / 2},\left\langle r^{2}\right\rangle_{n}^{1 / 2}$, and $\left\langle r^{2}\right\rangle_{m}^{1 / 2}$

\begin{tabular}{|c|c|c|c|c|c|c|c|}
\hline nucleus & $\begin{array}{c}\left\langle r^{2}\right\rangle_{c h}^{1 / 2} \\
(f m)\end{array}$ & $\begin{array}{c}\text { Exp. }\left\langle r^{2}\right\rangle_{c h}^{1 / 2} \\
(f m)\end{array}$ & $\begin{array}{c}\left\langle r^{2}\right\rangle_{p}^{1 / 2} \\
(f m)\end{array}$ & $\begin{array}{c}\text { Exp. } \\
\left\langle r^{2}\right\rangle_{p}^{1 / 2} \\
(f m)\end{array}$ & $\begin{array}{c}\left\langle r^{2}\right\rangle_{n}^{1 / 2} \\
(f m)\end{array}$ & $\begin{array}{c}\left\langle r^{2}\right\rangle_{m}^{1 / 2} \\
(f m)\end{array}$ & Exp. $\left\langle r^{2}\right\rangle_{m}^{1 / 2}(f m)$ \\
\hline${ }_{4}^{11} B e_{7}$ & 2.46 & $\begin{array}{c}2.463 \pm 0.016 \\
{[34]}\end{array}$ & 2.328 & $\begin{array}{c}2.361 \\
\pm 0.017 \\
{[34]}\end{array}$ & 3.253 & 2.95 & $2.91 \pm 0.05[34]$ \\
\hline${ }_{6}^{19} C_{13}$ & 2.528 & - & 2.40 & $\begin{array}{c}2.4(3) \\
{[13]}\end{array}$ & 3.455 & 3.16 & $3.16(7)[13]$ \\
\hline${ }_{3}^{11} L i_{8}$ & 2.47 & $\begin{array}{c}2.47 \pm 0.04 \\
{[34]}\end{array}$ & 2.338 & $\begin{array}{c}2.37 \pm 0.04 \\
{[34]}\end{array}$ & 3.865 & 3.515 & $3.5 \pm 0.09[34]$ \\
\hline
\end{tabular}
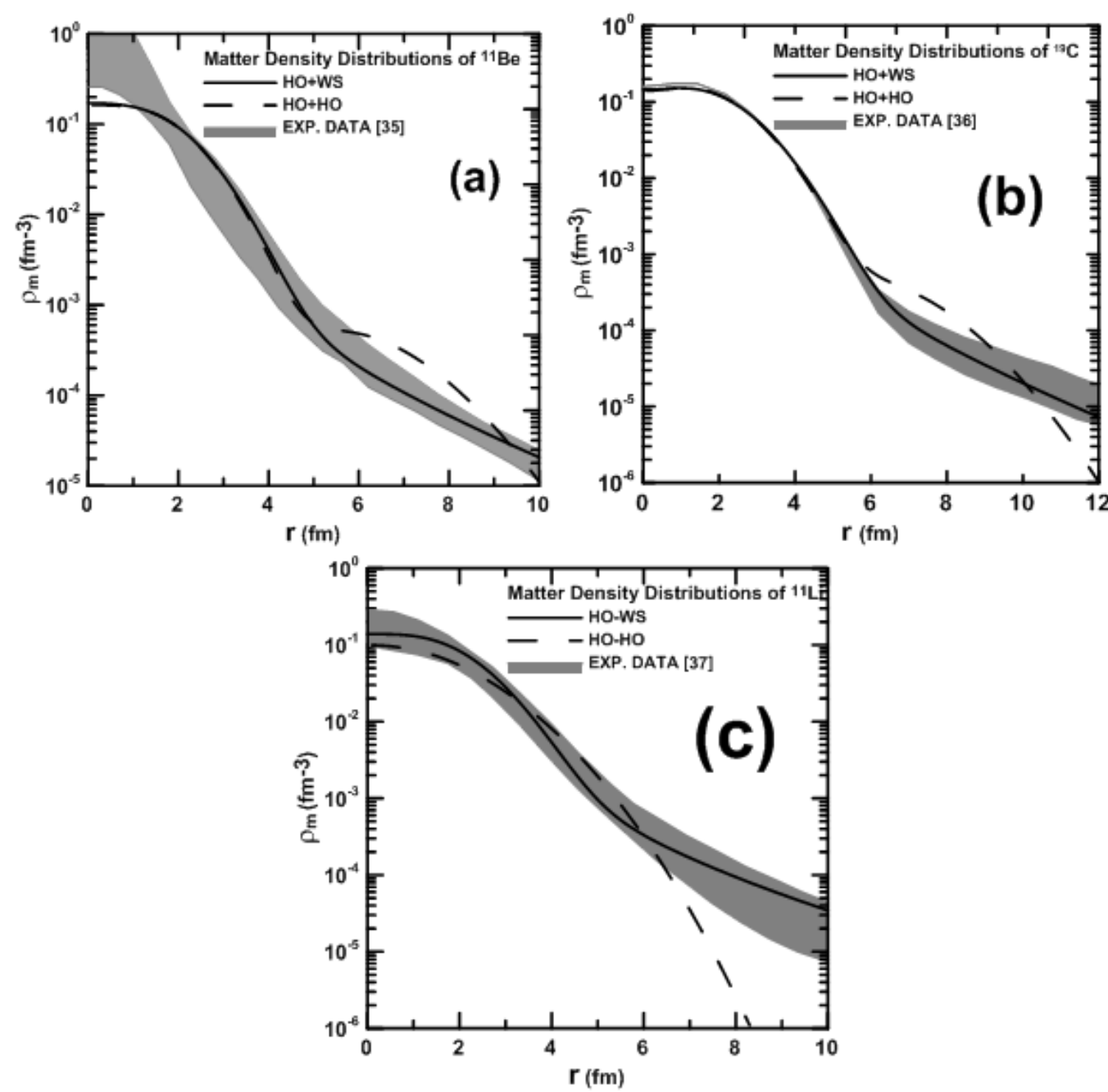

Fig. 1: The calculated matter density distributions for ${ }^{11} \mathrm{Be},{ }^{19} \mathrm{C}$ and ${ }^{11} \mathrm{Li}$.

The calculated charge form factors are illustrated in Fig. 2(a) for ${ }^{11} \mathrm{Be}, 2$ (b) for ${ }^{19} \mathrm{C}$ and $2(\mathrm{c})$ for ${ }^{11} \mathrm{Li}$, respectively, and compared with experimental data of the corresponding stable nuclei. In Fig. 2(a) and Fig. 2(b), the solid and dashed curves represent the calculated charge form factor in $\mathrm{HO}+\mathrm{WS}$ and
$\mathrm{HO}+\mathrm{HO}$, respectively. It is clear from aforementioned both figures that the results of $\mathrm{HO}+\mathrm{WS}$ shift the results upwards to approach experimental data, on contrary to the results of $\mathrm{HO}+\mathrm{HO}$ which underestimate the calculated charge form factors. In Fig. 2(c), the solid and dashed curves 
represent the calculated charge form factor using Tassie and BohrMottelson models, respectively where the incoherent sum of the $C 0$ and $C 2$ components are applied on ${ }^{11} \mathrm{Li}$ according to the Eq. (26). All results in Fig. 2 provide predictions for the future experiments on the electronradioactive beam colliders where the effect of the neutron and proton halo or skin on the charge density distributions and charge form factors is planned to be studied, where the normalization constant in Eq. (23) and (24) are computed using the CDD of $\mathrm{HO}+\mathrm{WS}$ so as to reproduce the experimental quadrupole moments, $-3.33 \pm 0.05$ ef $\mathrm{m}^{2}$ [38].
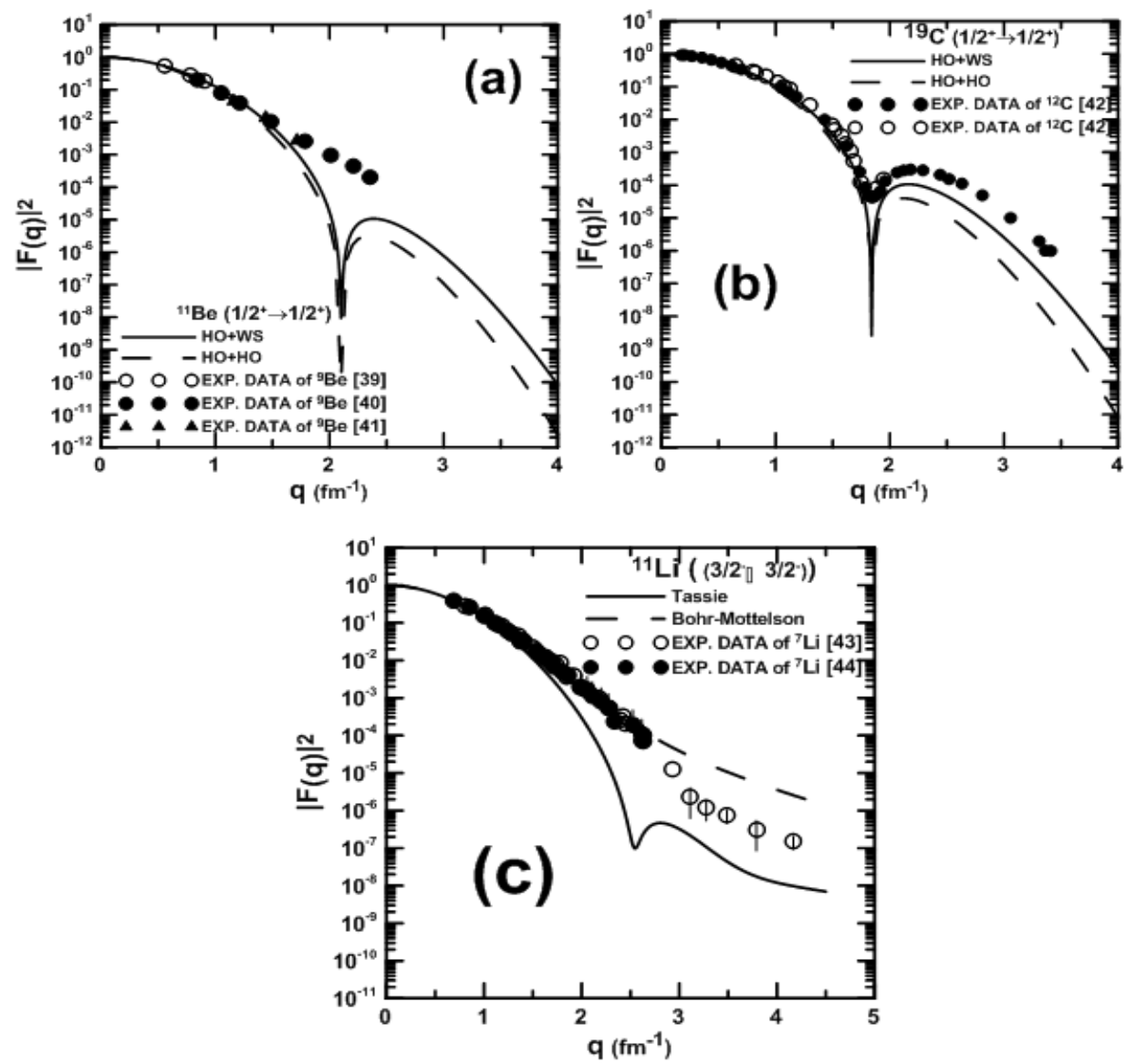

Fig. 2: The calculated charge form factors for halo ${ }^{11} \mathrm{Be},{ }^{19} \mathrm{C}$ and ${ }^{11} \mathrm{Li}$ nuclei compared with stable ${ }^{9} \mathrm{Be},{ }^{12} \mathrm{C}$ and ${ }^{7}$ Li nuclei.

\section{Conclusions}

The MDDs of one-neutron halo in ${ }^{11} \mathrm{Be}$ and ${ }^{19} \mathrm{C}$, and two-neutron halo in ${ }^{11} \mathrm{Li}$ have been calculated in $\mathrm{HO}$ for core parts and WS potential for halo parts. The elastic Coulomb electron scattering form factors for aforementioned nuclei have been studied in shell model. Besides, the quadrupole moment for ${ }_{3}^{11} L i_{8}$ are investigated in this work. A very good agreement with experimental data for the calculated $\mathrm{rms}$ charge and matter radii and MDDs are obtained for the three nuclei under study. The results of the calculated elastic charge form factors for the nuclei are controversial till future experiments on the electronradioactive beam colliders are settled. 


\section{References}

[1] I. Tanihata, H. Hamagaki, O. Hashimoto, S. Nagamiya, Y. Shida, N. Yoshikawa, O. Yamakawa, K. Sugimoto, T. Kobayashi, D.E. Greiner, N. Takahashi, Y. Nojiri, Phys. Lett., B 160 (1985) 380-384.

[2] I. Tanihata, H. Hamagaki, O. Hashimoto, Y. Shida, N. Yoshikawa, K. Sugimoto, O. Yamakawa, T. Kobayashi, N. Takahashi, Phys. Rev. Lett., 55 (1985) 2676-2679.

[3] P.G. Hansen, A.S. Jensen, B. Jonson, Annu. Rev. Nucl. Part. Sci., 45 (1995) 591-634.

[4] I. Tanihata, J. Phys., G 22 (1996) 157-198.

[5] B. Jonson, Phys. Rep., 389 (2004) 1-59.

[6] G. Baur, K. Hencken, D. Trautmann, Prog. Part. Nucl. Phys., 51 (2003) 487-564.

[7] P.G. Hansen, B. Jonson, Europhys. Lett., 4 (1987) 409-414.

[8] C. A. Bertulani. Journal of Physics G: Nuclear and Particle Physics, 34, (2007) 315-333.

[9] J. S. Al-Khalili and J. A. Tostevin, Phys. Rev. Lett., 76 (1996) 3903-3906.

[10] J. S. Al-Khalili, J. A. Tostevin, I. J. Thompson, Phys. Rev., C54 (1996) 1843-1852.

[11] H. Esbensen, K. Hagino, P. Mueller, H. Sagawa, Phys. Rev., C76 (2007) 1-6.

[12] G. D. Alkhazov, V. V. Sarantsev, Physics of Atomic Nuclei, 75 (2012) 1544-1549.

[13] R. Kanungo, W. Horiuchi, G. Hagen, G. R. Jansen, P. Navratil, F. Ameil, J. Atkinson, Y. Ayyad, D.Cortina-Gil, I. Dillmann, A. Estrade, A. Evdokimov, F. Farinon, H. Geissel, G. Guastalla, R.Janik, M. Kimura, R. Knobel, J. Kurcewicz, Yu. A. Litvinov, M. Marta, M. Mostazo, I. Mukha, C.Nociforo, H.J. Ong, S. Pietri, A. Prochazka, C. Scheidenberger, B. Sitar, P. Strmen, Y. Suzuki,M. Takechi, J. Tanaka, I. Tanihata, S.
Terashima, J. Vargas, H. Weick, J. S. Win_eld. Phys. Rev. Lett., 117 (2016) 1-5.

[14] Karataglidis S., Amos K., Fraser P., Canton L. and Svenne J. P., Nuclear Physics, A 813 (2008) 235-251.

[15] A. K. Hamoudi, R. A. Radhi, A. R. Ridha, Iraqi Journal of Physics, 13 (2015) 68-81.

[16] A. P. J. Brussard and P. W. M. Glademans, "Shell-model Application in Nuclear Spectroscopy", NorthHolland Publishing Company, Amsterdam (1977).

[17] B. A. Brown, S. E. Massen, P. E. Hodgson, Journal of Physics, G. 5 (1979) 1655-1698.

[18] L. R. B. Elton and A. Swift, Nuclear Physics, A 94 (1967) 52-72.

[19] S. Gamba, G. Ricco, G. Rottigni, Nuclear Physics, A 213 (1973) 383396.

[20] Peter Ring and Peter Schuck, "The Nuclear Many-Body Problem", Springer-Verlag (1981).

[21] T. DeForest Jr., J. D. Walecka, Advances in Physics, 15 (1966) 1-109.

[22] B. A. Brown, B. H. Wildenthal, C. F. Williamson, F. N. Rad, S. Kowalski, H. Crannell, J. T. O'Brien, Physical Review, C 32 (1985) 1127-1145.

[23] R. A. Radhi, A. K. Hamoudi, Z. A. Salman, Iraqi Journal of Physics, 2, 2 (2003) 11-18.

[24] Xiangdong Ji, B. H. Wildenthal, Phys. Riv., C38 (1988) 2849-2859.

[25] B. A. Brown, A. Arima, J. B. McGrory, Nuclear Physics, A277 (1977) 77-108.

[26] G. Bertsch and S. F. Tsai, Phys. Lett., B. 50 (1974) 319-322.

[27] H. Ui and T. Tsukamoto, Prog. Theor. Phys., 51 (1974) 1377-1386.

[28] Aage Bohr and Ben R. Mottelson, "Nuclear Structure Vol II", World Scientific (1998).

[29] B. A. Brown, R. Radhi, B. H. Wildenthal, Phys. Rep., 101 (1983) 313-358. 
[30] H. Chandra, G. Sauer, Phys. Rev. C13 (1975) 245-252.

[31] B. A. Brown, W. D. M. Rae, MSU-NSCL Report, (2007) 1-29.

[32] D. J. Millener and D. Kurath, Nuclear Physics, A 255 (1975) 315338.

[33] M. Wang, G. Audi, A.H. Wapstra, F.G.Kondev, M.MacCormic,

$\mathrm{X}$. Xu, B. Pfeiffer Chinese Physics, C36 (2012) 1603-2014.

[34] Isao Tanihata, Herve Savajols, Rituparna Kanungo, Progress in Particle and Nuclear Physics, 68 (2013) 215-313.

[35] M. Fukuda, T. Ichihara, N. Inabe, T. Kubo, H. Kumagai, T. Nakagawa, Y. Yano, I. Tanihata, M. Adachi, K. Asahi, M. Kouguchi, M. Ishihara, H. Sagawa, S. Shimoura, Phys. Lett., B. 268 (1991) 339-344.

[36] A. Ozawa, O. Bochkarev, L. Chulkov, D. Cortina, H. Geissel, M. Hellström, M. Ivanov, R. Janik, K. Kimura, T. Kobayashi, A. A. Korsheninnikov, G. Münzenberg, F. Nickel, Y.Ogawa, A.A. Ogloblin, M. Pfützner, V. Pribora, H. Simon, B. Sitár, P. Strmen, K. Sümmerer, T. Suzuki, I. Tanihata, M. Winkler, K. Yoshida, Nuclear Physics, A 691 (2001) 599-617.

[37] P. Egelhof, G.D. Alkhazov, M.N. Andronenko, A. Bauchet, A.V. Dobrovolsky, S. Fritz,
G.E. Gavrilov, H. Geissel, C. Gross, A.V. Khanzadeev, G.A. Korolev, G. Kraus, A.A. Lobodenko, G. Münzenberg, M. Mutterer, S.R. Neumaier, T. Schäfer, C. Scheidenberger, D.M. Seliverstov, N.A. Timofeev, A.A. Vorobyov, V.I. Yatsoura, Eur. Phys. J., A 15 (2002) 27-33.

[38] N. J. Stone. IAEA, INDC (NDS)0658, Distr. ND, February 2014,

'Table of nuclear magnetic dipole and electric quadrupole moments'.

[39] J. P. Glickman, W. Bertozzi, T. N. Buti, S. Dixit, F. W. Hersman, C. E. Hyde-Wright, M. V. Hynes, R. W. Lourie, B. E. Norum, J. J. Kelly, B. L. Berman, D. J. Millene, Phys. Rev., C 43 (1991) 1740-1757.

[40] J. A. Jansen, R. Peerdeman, C. De Vries, Nucl. Phys., A188 (1972) 337352.

[41] M. Bernheim, T. Stovall, D. Vinciguerra, Nucl. Phys., A97 (1967) 488-504.

[42] I. Sick, J.S. McCarthy, Nuclear Physics, A 150 (1970) 631-654.

[43] L. R. Suelzle, M. R. Yearian, H. Crannell, Phys. Rev., 162 (1967) 9921005.

[44] J. Lichtenstadt, J. Alster, M.A. Moinester, J. Dubach, R.S. Hicks, G.A. Peterson, S. Kowalski. Phys. Lett., B. 219 (1989) 394-398. 